Annuaire du Collège de France 2017-2018

\title{
Physique quantique
}

Jean-Michel Raimond

\section{OpenEdition}

Journals

Édition électronique

URL : https://journals.openedition.org/annuaire-cdf/16214

DOI : 10.4000/annuaire-cdf. 16214

ISBN : 978-2-7226-0572-5

ISSN : 2109-9227

Éditeur

Collège de France

Édition imprimée

Date de publication : 30 décembre 2020

Pagination : 678-680

ISBN : 978-2-7226-0516-9

ISSN : 0069-5580

Référence électronique

Jean-Michel Raimond, «Physique quantique », L'annuaire du Collège de France [En ligne], 118 | 2020

mis en ligne le 01 avril 2021, consulté le 22 août 2022. URL : http://journals.openedition.org/annuairecdf/16214 ; DOI : https://doi.org/10.4000/annuaire-cdf.16214 
ZHu Z., FAuqué B., BehNiA K. et FusEYA Y., « Magnetoresistance and valley degree of freedom in bulk bismuth », Journal of Physics: Condensed Matter, vol. 30, $\mathrm{n}^{\mathrm{O}}$ 31, 2018, 313001, DOI : 10.1088/1361-648X/aaced7 [arXiv: 1801.07098].

Collignon C., Lin X., Rischau C.W., Fauqué B. et Behnia K., « Metallicity and superconductivity in doped strontium titanate », Annual Review of Condensed Matter Physics, vol. $10, \mathrm{n}^{\mathrm{o}}$ 1, 2019, p. 25-44, DOI : 10.1146/annurev-conmatphys-031218-013144 [arXiv: 1804.07067].

\section{RECHERCHES SUR LA PHYSIQUE MÉSOSCOPIQUE À PARTIR DE GRAPHĖNE ET DE JONCTIONS JOSEPHSON / JOSEPHSON JUNCTION SPECTROSCOPY OF MESOSCOPIC SYSTEMS}

Responsable : Çağlar GiRiT

\section{RECHERCHE}

Page web : https://www.college-de-france.fr/site/young-team-incubator/ Presentation_2.htm.

\section{PHOTONIQUE QUANTIQUE / QUANTUM PHOTONICS}

Responsable : Alexei OURJOUMTSEV

\section{RECHERCHE}

Page web : https://www.college-de-france.fr/site/young-team-incubator/ Presentation_3.htm.

\section{PHYSIQUE QUANTIQUE}

Responsable : Jean-Michel RAIMOND

\section{RECHERCHE}

Page web : https://www.college-de-france.fr/site/physique-quantique/Presentation.htm.

Notre activité s'est orientée en 2017-2018 vers trois directions principales : jeux quantiques dans les multiplicités de Rydberg; électrodynamique quantique en cavité ; simulation quantique avec les atomes de Rydberg. 
Nous avons obtenu depuis 2016 des résultats importants dans la première direction, soutenue par un contrat ANR jeune (PI : S. Gleyzes) et un ITN européen (QUSCO). Nous avons en particulier observé l'oscillation de Rabi cohérente entre un niveau de faible moment angulaire et le niveau circulaire de moment angulaire maximum. Nous avons réalisé un électromètre fondé sur des états «chat de Schrödinger », avec une sensibilité au-delà de la limite quantique standard.

Nous avons récemment étendu le champ d'application de cet électromètre. En manipulant temporellement le dipôle électrique de l'atome, nous mesurons directement la fonction de corrélation temporelle du champ, avec une bande passante de $5 \mathrm{MHz}$. Nous pouvons ainsi reconstruire complètement les propriétés statistiques d'un champ stochastique. Ce résultat ouvre des voies prometteuses pour la mesure de signaux aléatoires en physique mésoscopique. Un article a été soumis.

Nous avons réalisé un magnétomètre, fondé sur une superposition des deux niveaux circulaires de moments angulaires opposés. Dans une image classique, l'électron tourne sur l'orbite circulaire du modèle de Bohr dans les deux sens en même temps. Un seul des atomes dans cet état «chat de Schrödinger » fournit une mesure du champ magnétique aussi sensible que celle de 1000 atomes dans un magnétomètre conventionnel. Ces résultats ont été publiés depuis dans la revue Nature Physics.

Enfin, en étroite collaboration avec le groupe de Christiane Koch (Univ. Kassel), nous appliquons des techniques de contrôle quantique pour améliorer considérablement la préparation des atomes circulaires à partir de niveaux accessibles par laser, en évitant les imperfections liées aux défauts quantiques de niveaux de faible moment angulaire. Nous avons pu ainsi préparer, avec les impulsions radiofréquence calculées par le groupe de Kassel, des niveaux circulaires avec une fidélité de $95 \%$ en 120 ns, un résultat sans précédent. Nous pouvons concevoir des impulsions préparant des états plus complexes, comme les superpositions circulaires/état de grand dipôle au cœur de notre électromètre. Ces résultats sont en cours de rédaction.

Dans la première de nos expériences d'électrodynamique quantique en cavité, un atome traversant deux cavités intrique leur état. Nous avons réalisé une superposition où un photon est dans une cavité ou dans l'autre, et nous développons en collaboration avec P. Rouchon (Mines-Paristech) une méthode efficace de reconstruction de l'état quantique joint des deux cavités. Les résultats de cette reconstruction sont soumis. Nous allons étendre notre activité à des expériences de thermodynamique quantique (soutenues par un contrat ANR QUDICE - PI : Igor Dotsenko), où nous utiliserons les atomes pour échanger travail et chaleur entre les deux cavités.

En tirant parti du grand temps d'interaction entre des atomes de Rydberg lents et une cavité, nous avons résolu par spectroscopie micro-onde la transition d'un atome en présence d'un nombre donné de photons. Nous sélectionnons ainsi un nombre de photons donné en détectant un seul atome. En modulant dans le temps la fréquence de l'atome, nous travaillons à préparer des états plus complexes, superpositions de deux états de Fock différents, par exemple. Un premier papier sur ces résultats est soumis.

Notre dernière activité doit devenir notre principale ligne d'investigation dans un futur proche. Il s'agit de la réalisation d'un simulateur quantique de chaîne (ou de réseaux) de spins. Elle est soutenue par un contrat QUANTERA (ERYQSENS), par une ANR (TRYAQS), une action du réseau SIRTEQ (CARAQUES), une action du 
Flagship sur les technologies quantiques (PASQUANS) et, enfin, par un contrat ERC Advanced (TRENSCRYBE) porté par M. Brune qui prendra la direction de l'équipe au $1^{\text {er }}$ janvier 2019. Nous envisageons de piéger par laser des atomes de Rydberg circulaires dans un condensateur plan qui inhibe leur émission spontanée. La durée de vie des atomes peut atteindre une minute, un résultat remarquable. Nous proposons une méthode déterministe de préparation et de détection d'une chaîne contenant jusqu'à une quarantaine d'atomes. Les interactions dipolaires entre ces atomes, un habillage micro-onde et un choix optimal de niveaux rendent la chaîne équivalente à un ensemble de spins interagissant entre plus proches voisins de la façon la plus générale, avec des temps caractéristiques très courts à l'échelle du temps de vie de la chaîne. Sa dynamique simule donc un des systèmes paradigmatiques de la physique de la matière condensée, suffisamment complexe pour qu'une approche numérique classique soit difficile dans le cas général en raison de la taille gigantesque de l'espace de Hilbert. Tous les paramètres du Hamiltonien peuvent être contrôlés en temps réel, ouvrant des perspectives nouvelles pour la simulation quantique. Un article important résumant la proposition a été publié. Du point de vue expérimental, nous travaillons sur la première expérience de piégeage d'un atome circulaire. Nous avons conçu une expérience de pinces optiques, qui nous permettra très rapidement d'étudier l'interaction entre atomes circulaires piégés.

\section{PUBLICATIONS}

Patsch S., Reich D.M., Raimond J.-M., Brune M., Gleyzes S. et Koch C.P., «Fast and accurate circularization of a Rydberg atom », Physical Review A, vol. 97, no 5, 2018, 053418, DOI : 10.1103/PhysRevA.97.053418 [arXiv: 1802.09504].

Nguyen T.L., Raimond J.M., SAYrin C., Cortiñas R., Cantat-Moltrecht T., Assemat F., Dotsenko I., Gleyzes S., Haroche S., Roux G., Jolicoeur T. et Brune M., «Towards quantum simulation with circular Rydberg atoms », Physical Review X, vol. 8, n 1, 2018, 011032, DOI : 10.1103/PhysRevX.8.011032 [arXiv: 1707.04397].

Dietsche E.K., LARrouy A., RAimond J.M., Brune M. et GleyZeS S., « Quantum-enabled electrometer measures field transients and correlation function », 2018, http://arxiv.org/ abs/1805.06870. 